\title{
Research on Optical Communication System based on Dispersion Compensation Fiber and Nonlinear Effect
}

\author{
ZHANG Yajuan ${ }^{1, a}$ \\ ${ }^{1}$ Department of Electronics and telecommunication Engineering, Wuhan Polytechnic, Wuhan \\ 430074, China \\ azhangyajuan@126.com
}

\begin{abstract}
Keywords: Optical Communication System, Nonlinear Optical Effect, Dispersion Compensation Fiber
\end{abstract}

\begin{abstract}
In this paper, we have analyzed and described dark-Gaussian soliton collision behaviors within a modified add/drop multiplexer system consisting of one center ring and one smaller ring on the left side. Optical communication system based on dispersion compensation fiber and nonlinear optical effect is taken into consideration. The results show that, the full width of half peak multiplexing signal and FSR of $0.8 \mathrm{~nm}$ and $5 \mathrm{~nm}$ potential, the generation of Wells can be the logic code, in optical soliton pulse of light and dark and FSR fhhm $0.54 \mathrm{~nm}$ and $4.71 \mathrm{~nm}$, can be obtained. Further potential research areas are discusses in the final parts.
\end{abstract}

\section{Introduction}

Nonlinear effects and dispersion in optical fibers have become an area of academic research and of great importance in the optical fiber based communication systems. The nonlinear effect and dispersion effect the performance of optical fiber communication system of communication between the two at the receiving end. Considering the influence of nonlinear and dispersion, the change of the situation. In some cases, the nonlinear can offset the dispersion [1-5]. Out of the several techniques for dispersion compensation, DCF is the most widely deployed dispersion compensator, as they are cascadable, commercially available and compatible with all optical network concepts [6-8].Inside each DCF span, the pulses of a longer wavelength channel travel faster than pulses of a shorter wavelength channel, whereas the opposite is the case inside each SMF span [9] .The condition for perfect dispersion compensation for a pulse to regain its original shape, is given by D1L1 + D2L2 $=0$, where D1 and D2 are dispersion coefficients of SMF and DCF, L1 and L2 are the corresponding length respectively. For practical reasons, L2 should be as small as possible [10-11]. The main optical fiber nonlinear effects in standard SMF the self-phase modulation (SPM), which is caused by the nonlinear refractive index of the dependence pulse strength [3]. Deepak gupta etal, choose appropriate waveforms and pulse power, SPM and GVD (gnu visual debugger) can compensate for each other.

It is known that fiber has lowest loss at 1550nm and also that efficient amplifiers operate around this wavelength. So it is desirable to operate around $1550 \mathrm{~nm}$ [2].With increased bitrates it has been shown that Return-to-Zero (RZ) modulation formats offer certain advantages over NRZ, as they tend to be more robust against distortions [9]. Therefore, a TDMA system can be realized as a channel access method for shared medium networks, where the users receive information with different time slots. This allows multiple stations to share the same transmission medium while using only a part of its channel capacity. TDMA can be used in digital mobile communications and satellite systems. Thus, in the TDMA system, instead of having one transmitter connected to one receiver, there are multiple transmitters, where high-secured signals of quantum codes along the users can be transmitted. To date quantum code is the only form of information that can provide the perfect communication security. Dark- Gaussian soliton controls within a semiconductor add/drop multiplexer has numerous applications. In the following captures, we will discuss the issues. 


\section{Proposed Methodology}

OPTSIM is advanced optical communication system simulation packages which can be used to design optical. Communication systems and simulate them to determine their performance given various component parameters. To guarantee the highest possible accuracy and real-world result, OPTSIM simulation software is used for the work. Input optical field of dark soliton and Gaussian pulse are introduced into the input and add ports of the proposed add/drop interferometer system respectively, shown in Fig. 1.

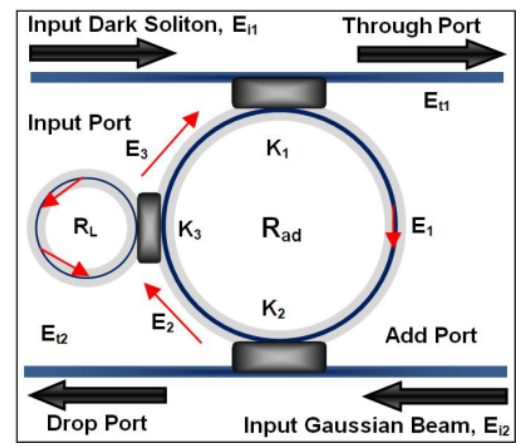

Figure 1: The Diagram of an Add/drop Interferometer System

The System Description. The simulation setup is shown in the Fig.2. This system consists of three major sections i.e., transmitter, fiber section and receiver section.

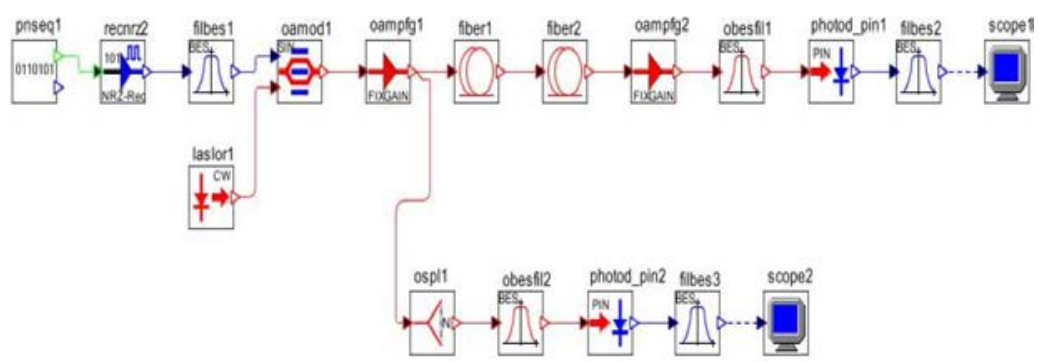

Figure 2: The Simulation Set Up With NRZ Rectangular Driver

The transmitter consists of pseudorandom bit sequence generators, which generate bit sequence, which is fed to coder to produce an electrical coded signal. The pulses, after passing through a low pass filter are then modulated with continuous wave (CW) laser radiation of wavelength $1550 \mathrm{~nm}$ to obtain optical pulses .Mach Zender Modulator is used as external modulator to obtain optical pulses from electrically coded signal. PRBS has launched 10Gbps rate. Analysis of NRZ and RZ code encoder by using the influence of different modulation schemes is vital. Here the use of the light source is a continuous wave laser with the Lorenz center frequency of emission of the 193.4thz center, power emission wavelengths were 1dB 1547-1560 CW. Electronic low-pass filter using the Bessel filter. Optical fiber section consists of single mode fibers and dispersion compensated fibers in the channel. The optical signal after boosting is fed into the single mode fiber. The $100 \mathrm{~km}$ fiber span consists of two segments, SMF of length L1 km and DCF of length L2km. L1 and L2are kept variables. Fixed gain amplifiers are used as boosters and preamplifiers. Preamplifier gain is fixed as $30 \mathrm{~dB}$ with figure of merit 4.Gain of booster is varied from $10 \mathrm{~dB}$ to $24 \mathrm{~dB}$. For the design of communication channels, the parameters of SMF and DCF are as shown in the figure 3. Optical receiver is composed of PIN diode is used as the photoelectric detector, optical filter for Lorenzian band pass filter, Bessel low pass filter electric filter.pin photodiode converts the light signal into the electrical signal of the Bessel filter is provided with a type of modulation waveforms for determining the cut-off frequency.. Finally, a visualization tool called range, used by the OPTSIM to display the results in the output. It is a kind of optical or electronic oscilloscope for large data processing options, eye display characteristics and BER estimation.

Pulse Analysis. A system known as optical multiplexer can be used to increase the channel capacity and security of the signals, where the beam splitters generate high capacity of binary codes within the proposed system. Therefore, ring resonator system is used to form the optical potential 
wells. The multiplexed potential wells are formed and transmit via an available link, where the logic codes can be sent out with different time, used for high capacity transmission of the secured data. In this work narrow pulses with FHHM of $9.57 \mathrm{~nm}$ and $8 \mathrm{~nm}$ could be obtained from the drop and through ports of the add/drop interferometer system respectively. The outputs of different center wavelengths are combined and used to generate multiple potential well signals, where the multiple signals with FWHM and FSR of $0.8 \mathrm{~nm}$ and $5 \mathrm{~nm}$ could be obtained respectively.

\begin{tabular}{|c|c|c|c|}
\hline $\begin{array}{c}\text { Serial } \\
\text { number }\end{array}$ & Parameter & SNIF & DCF \\
\hline 1 & Filber length $(\mathbf{k m})$ & $100.75\left(\mathrm{~L}_{1}\right)$ & $0.25\left(\mathrm{~L}_{2}\right)$ \\
\hline 2 & $\begin{array}{l}\text { Fiber dispersion D } \\
(\mathrm{p} s / \mathrm{nm} / \mathrm{km})\end{array}$ & 17 & .95 \\
\hline 3 & $\begin{array}{l}\text { Effective come Area Aef } \\
\left(\mathrm{nm}^{2}\right)\end{array}$ & 80 & 20 \\
\hline 4 & 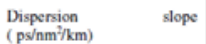 & 0.08 & -0.1 \\
\hline 5 & $\begin{array}{l}\text { Non lincar nefractive index } \\
\mathrm{n}_{3}\left(\mathrm{x} \times 10^{-5}\right)\end{array}$ & 2.5 & 2.5 \\
\hline 6 & Attenuation $\alpha(\mathrm{dB} / \mathrm{km})$ & 0.25 & 0.5 \\
\hline
\end{tabular}

Figure 3: The Parameter Of SMF and DCF

\section{The Experimental Simulation}

Set-up of the Experiment. The effect due to nonlinear dependence of the refractive index on pulse intensity and power (self-phase modulation) for different modulation schemes is analyzed. The degradation performance of fiber link is due to the booster amplifier nonlinear effect was observed by the presence of. For smaller DCF length was observed better performance, because in the anomalous dispersion region, the SPM offset dispersion.

Variation Of BER with Bit Rate. The bit rate of the signal is varied in PRBS generator and corresponding values of BER are noted. The plot of BER with respect to bit rate is shown in fig. 4 . Figure 4 shows the variation of BER bit rate, where L1 and l2are are $88 \mathrm{~km}$ and $12 \mathrm{~km}$ respectively. Our simulation, allowing Ye observed maximum bit rate of 10Gbps. Experiments repeated booster gain of $10 \mathrm{~dB}, 16 \mathrm{~dB}$ and $22 \mathrm{~dB}$, the starting point of the downward movement of the flat region.

The Variation Of BER and Q with DCF Length. Fig. 5 shows the variation of BER with DCF length for booster gain 10dB. The variation of Q value with DCF length is shown in fig. 6.

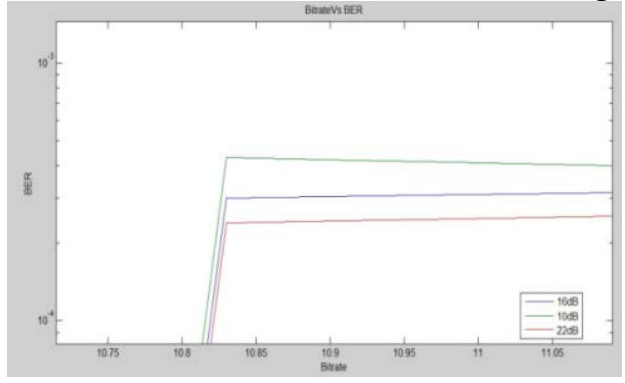

Figure 4: The Variation of BER

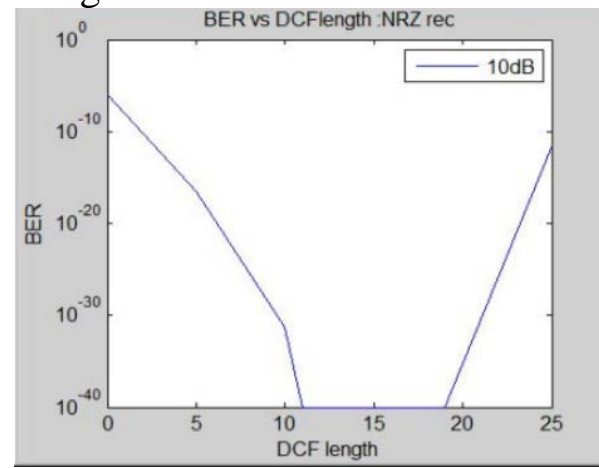

Figure 5: The Variation of BER with DCF Length 


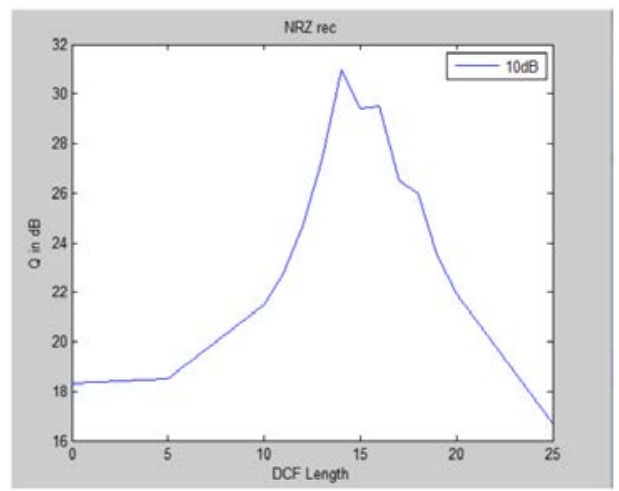

Figure 6: The Variation Of Q Factor with DCF Length

The best eye diagrams obtained for different booster gain are shown in fig.7.
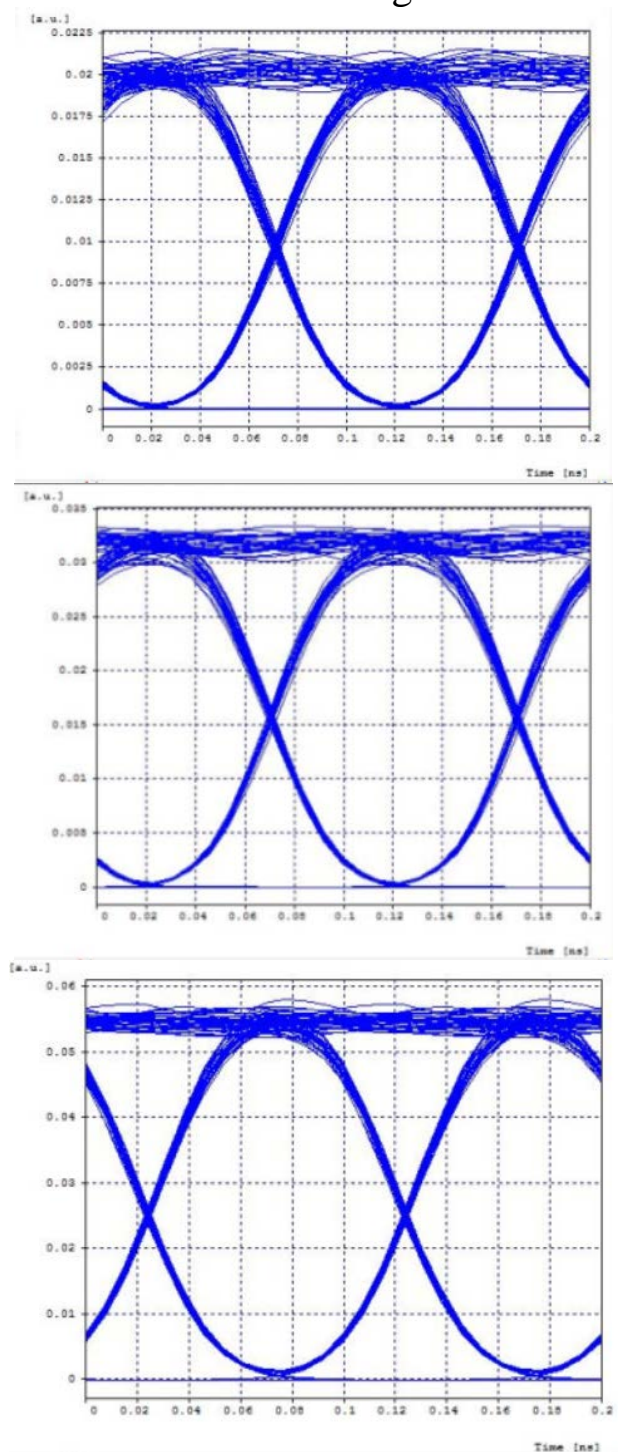

Figure 7: The Eye Diagram

The Observations with Different Modulation Schemes. In fig.8, the performances of the dispersion compensated fiber optic link with different modulation schemes are shown. Comparing Fig.5 and Fig.7 (a) the following observations are noted. When NRZ raised cosine driver is used, DCF minimum value with BER minimum is constant within the range of different gain values in our study. It was observed that for a specific gain value and DCF length, the BER is found to be less for NRZ rectangular driver. 


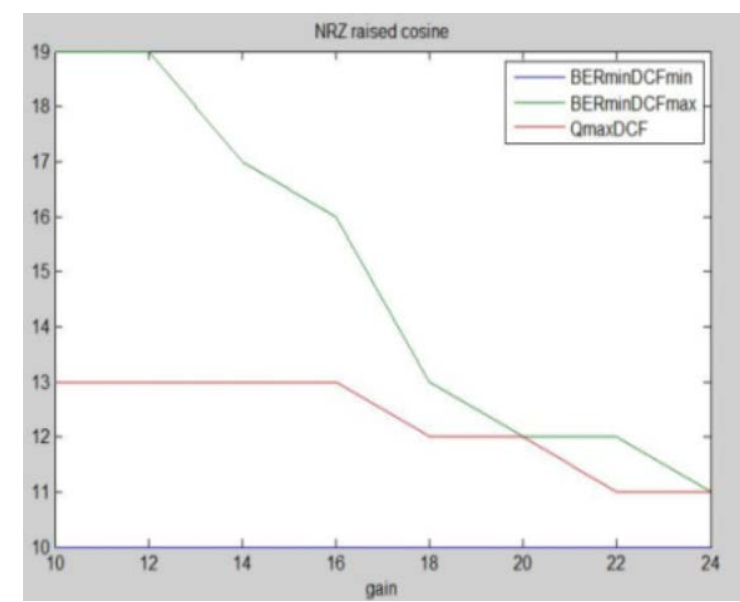

Figure 8: The Variation of BERmin

\section{Conclusions}

We have analyzed and described dark-Gaussian soliton collision behaviors within a modified add/drop multiplexer system consisting of one center ring and one smaller ring on the left side. In the formation of trap optical tweezers can generate and use PBS for binary code. We propose a quantum logic based on the code in the data communication capacity through the use of optical network link code is reasonable network security interesting concept. The required binary code can be introduced in a potential well generated signal. Wells's potential is very fixed optical signal because of its central wavelength, such as signal detection is very difficult low strength. Therefore, in the significance of the study, potential Wells signal of fixed height in the MRRS system, in the part of the system, is composed of a transmission part can be used for quantum encryption transmission. The results show that, the full width of half peak multiplexing signal and FSR of 0.8 $\mathrm{nm}$ and $5 \mathrm{~nm}$ potential, the generation of Wells can be the logic code, in optical soliton pulse of light and dark and FSR fhhm $0.54 \mathrm{~nm}$ and $4.71 \mathrm{~nm}$, can be obtained. The experimental result shows the effectiveness of the proposed method. In the future, we plan to do more research to modify our model.

\section{References}

[1] Cheng, Jingchi, et al. "Relative phase noise estimation and mitigation in Raman amplified coherent optical communication system." Optics express 22.2 (2014): 1257-1266.

[2] Amiri, I. S., et al. "Digital binary codes transmission via TDMA networks communication system using dark and bright optical soliton." Journal on Computing (JoC) 2.1 (2014).

[3] Roberts, Kim B., Maurice S. O'sullivan, and Leo L. Strawczynski. "Monitoring phase nonlinearities in an optical communication system." U.S. Patent No. 8,781,317. 15 Jul. 2014.

[4] Abaza, Mohamed, et al. "Diversity techniques for a free-space optical communication system in correlated log-normal channels." Optical Engineering 53.1 (2014): 016102-016102.

[5] Walker, Brian, et al. "Addressing channel noise and bit rate in a multi-channel free space optical communication system." SPIE Defense+ Security. International Society for Optics and Photonics, 2014.

[6] Amiri, I. S., et al. "Generation of quantum photon information using extremely narrow optical tweezers for computer network communication." Journal on Computing (JoC) 2.1 (2014).

[7] Shrivastava, Alankrati, and Manish Saxena. "Analysis of Optical Communication System for Compensation of Dispersion by Comparing using Fiber Bragg Grating." (2014).

[8] Ogasahara, Daisaku. "Signal processing circuit, signal processing method, optical receiver and optical communication system." U.S. Patent No. 8,705,987. 22 Apr. 2014. 
[9] Balakrishnan, Alampallam V., ed. Advances in communication systems: theory and applications. Vol. 2. Academic Press, 2014.

[10] Yang, Fan, Julian Cheng, and Theodoros A. Tsiftsis. "Free-space optical communication with nonzero boresight pointing errors." Communications, IEEE Transactions on 62.2 (2014): 713-725.

[11] Chi, Nan, et al. "Ultra-high-speed single red-green-blue light-emitting diode-based visible light communication system utilizing advanced modulation formats." Chinese Optics Letters 12.1 (2014): 010605. 